\title{
NOTA TECNICA \\ Efecto del árbol madre sobre la rizogénesis de Nothofagus alessandrii
}

\author{
Parental tree effects on rhizogenesis in Nothofagus alessandrii
}

\author{
ROMULO SANTELICES
}

Universidad Católica del Maule, Facultad de Ciencias Agrarias y Forestales, Centro de Investigación en Biotecnología Silvoagrícola, Casilla 617, Talca, Chile. e-mail rsanteli@ucm.cl

\begin{abstract}
SUMMARY
The effect of parent tree on the rooting capacity of stem cuttings of Nothofagus alessandrii Espinosa was studied. Stem cuttings were collected from 20 trees of four years old on November 2002. The trial was carried out in a plastic covered greenhouse located in the forest nursery of the Universidad Catolica del Maule in Talca. In the same nursery the parent tree were also cultivated. Cuttings were treated with indolebutyric acid $(0.5 \%)$. The greenhouse was equipped with a mist system and bottom heating that maintained a constant temperature around of $21^{\circ} \mathrm{C}$ at the base of the stem cuttings. The substrate used was germ free sawdust of Pinus radiata D. Don. Each trial was kept under observation for 3 months. Results indicate that there is a strong influence of the parent tree on the rooting capacity of $N$. alessandrii. For each parent tree, the rooting capacity oscillated from 0 to $100 \%$, with an average of 2.6 roots of $5.8 \mathrm{~cm}$ length.
\end{abstract}

Key words: Nothofagus alessandrii (ruil); parent tree; rooting capacity; indolebutyric acid (IBA).

\section{RESUMEN}

Se estudió el efecto que tiene el árbol madre en el arraigamiento de estacas de tallo de Nothofagus alessandrii Espinosa. La cosecha se efectuó en 20 árboles de cuatro años de edad en el mes de noviembre de 2002. El ensayo se llevó a cabo en un invernadero cubierto por polietileno en el vivero de la Universidad Católica del Maule, ubicado en la ciudad de Talca, lugar donde también se cultivaron los árboles madre. Las estacas fueron tratadas con ácido indolbutírico al 0,5\%. Se contó con un sistema de riego automatizado y con camas calientes de arraigamiento que permitieron mantener en la base de las estacas una temperatura alrededor de los $21^{\circ} \mathrm{C}$. El substrato empleado consistió en aserrín de Pinus radiata D. Don, el que fue previamente hervido en agua durante al menos una hora para así eliminar eventuales toxinas y hongos. El ensayo se mantuvo en observación por un lapso de tres meses. Los resultados indican que hay una fuerte influencia del árbol madre de donde son cosechadas las estacas en el enraizamiento de $N$. alessandrii. El enraizamiento de cada árbol madre varió de 0 a $100 \%$, con un promedio de 2,6 raíces de $5,8 \mathrm{~cm}$ de longitud por estaca.

Palabras clave: Nothofagus alessandrii (ruil); árbol madre; capacidad de arraigamiento; ácido indolbutírico (AIB). 


\section{INTRODUCCION}

Nothofagus alessandrii Espinosa es una de las especies más interesantes del bosque nativo de la región mediterránea de Chile. Tiene características únicas desde el punto de vista ecológico y evolutivo $(1,2)$, y de acuerdo a la calidad de su madera podría tener proyecciones difíciles de dimensionar. Sin embargo, actualmente se encuentra en una delicada situación desde el punto de vista de su conservación, llegando a ser declarada oficialmente como en peligro de extinción (3). Hoy se encuentran poco más de 300 ha distribuidas en bosquetes muy fragmentados e inmersos en plantaciones de rápido crecimiento (4), todos regenerados por monte bajo (5). Los pocos bosques que hoy existen se encuentran degradados producto de la sobreexplotación a que han estado sujetos desde principios del siglo pasado.

Una forma de mejorar el estado de conservación es incrementando el recurso por medio del manejo. En consecuencia, es necesario desarrollar técnicas orientadas a la repoblación de las áreas afectadas y una primera etapa debiera estar dirigida a conocer las diferentes formas de propagación de estas especies. La propagación vegetativa por estacas tiene una serie de ventajas, donde sobresale la posibilidad de reproducir clones (6). Sin embargo, existe evidencia de que para algunas especies el proceso de arraigamiento está condicionado genéticamente (7) y que para $N$. alessandrii el árbol madre de donde se obtenga el material vegetal podría condicionar el arraigamiento (8).

Sobre la base de lo antes mencionado, el objetivo de este trabajo es realizar una investigación preliminar respecto de la influencia que tiene el árbol madre en el arraigamiento de estacas de $N$. alessandrii.

\section{MATERIAL Y METODOS}

El ensayo se llevó a cabo en la Universidad Católica del Maule, ubicada en la ciudad de Talca, Región del Maule. Se contó con un invernadero recubierto con polietileno, implementado con un sistema de camas calientes de arraigamiento, que permitió que el sustrato se mantuviera a una temperatura constante alrededor de $\operatorname{los} 21^{\circ} \mathrm{C}$. El sustrato utilizado fue aserrín de Pinus radiata D.
Don, el que previamente fue hervido en agua durante al menos una hora, con el propósito de eliminar eventuales toxinas y hongos.

El riego fue automatizado y para ello se contó con un equipo llamado mistamatic, que activa el sistema de riego en función de la humedad ambiental y, además, se contó con aspersores conocidos como microjet que se caracterizan por pulverizar las gotas de agua, emulando el riego de niebla. Se mantuvo una alta humedad ambiental, sobre el $60 \%$, y la frecuencia y cantidad de riego varió de acuerdo a las condiciones climáticas.

El material vegetal fue cosechado en la mañana del 15 de noviembre de 2002 antes de las 10 horas. La colecta se efectuó de 20 árboles madre de cuatro años de edad, propagados por vía sexual, cultivados en el mismo vivero, y de procedencia El Huelón (comuna de Curepto, provincia de Talca, $\left.35^{\circ} 05^{\prime} \mathrm{S}, 7^{\circ} 03^{\prime} \mathrm{O}\right)$. Por restricciones naturales en la cantidad de estacas por árbol (altura y cantidad de ramas), sólo fue posible cosechar cinco por cada uno, todas de la zona apical de las ramas. Las estacas se dimensionaron de $5 \mathrm{~cm}$ de longitud, tuvieron al menos tres yemas visibles y fueron homogéneas respecto a su diámetro. Los cortes se efectuaron en ángulo recto e inmediatamente después de que fueron separadas del árbol madre se instaló el ensayo.

Como medida preventiva se desinfectaron las estacas antes de instalarlas en las camas calientes de arraigamiento. Para ello se preparó una mezcla de $6 \mathrm{~g}$ de Strepto Plus y $18 \mathrm{~g}$ de Dithane M45 en 101 de agua y su aplicación se realizó con una bomba de espalda.

Las estacas fueron tratadas con ácido indolbutírico al $0,5 \%(\mathrm{p} / \mathrm{p})$ dispersado en talco inerte y para preparar la auxina se consideraron las recomendaciones de Santelices (9). Los esquejes fueron sumergidos en agua y luego en la solución de ácido indolbutírico en los primeros $2 \mathrm{~cm}$ basales, teniendo la precaución de que la auxina no quedara aglomerada. Luego se instalaron en las camas de arraigamiento hasta una profundidad de aproximadamente $2,5 \mathrm{~cm}$.

El ensayo se mantuvo en observación durante tres meses en las camas de arraigamiento. Al final de este período se evaluó la sobrevivencia y formación de callo de la estaca (independiente de si formó o no raíces), la capacidad de arraigamiento y la producción de raíces. 


\section{RESULTADOS Y DISCUSION}

Existe una marcada influencia del árbol madre en el proceso de rizogénesis en estacas de $N$. alessandrii (cuadro 1), no sólo en la gran diferencia en las tasas de arraigamiento observadas, sino que también en lo que se refiere a calidad de raíces.

Son variados los factores que condicionan el éxito en el proceso de arraigamiento, siendo la juvenilidad uno de los importantes para algunas especies de la familia Fagaceae (10). A medida que aumenta la edad del material a propagar, como regla general, debería disminuir la capacidad de enraizamiento $(6,10,11,12)$. En este caso, no podría atribuirse a este factor la nula o baja capacidad de arraigamiento que mostraron algunos clones porque su edad fue de cuatro años. Por lo general, la capacidad de enraizamiento disminuye después del quinto año de edad (11).

Es común que diversos clones de algunas especies presenten grandes diferencias en la capacidad de arraigamiento. Por ejemplo, en Eucalyptus camaldulensis Dehn se ha observado que con algunos se puede llegar a obtener sobre un $80 \%$ de enraizamiento, mientras que con otros no se consigue la inducción de raíces (13). Considerando que el $25 \%$ de los clones de $N$. alessandrii analizados tuvo una respuesta nula al enraizamiento, este factor debiera tenerse en cuenta al diseñar un método de propagación por esta vía, sobre todo si se intenta realizar algún tipo de mejoramiento.

La diferencia apreciada en la capacidad de arraigamiento de los diferentes clones concuerda

\section{CUADRO 1}

Efecto del árbol madre sobre la capacidad rizogénica de $N$. alessandrii.

Effect of parent tree in the rooting capacity of $N$. alessandrii.

\begin{tabular}{|c|c|c|c|c|}
\hline \multirow{2}{*}{$\begin{array}{l}\text { Árbol } \\
\text { madre }\end{array}$} & \multirow{2}{*}{$\begin{array}{l}\text { Formación } \\
\text { de callo } \\
\qquad \%)\end{array}$} & \multirow{2}{*}{$\begin{array}{l}\text { Sobrevivencia y } \\
\text { arraigamiento } \\
\text { (\%) }\end{array}$} & \multicolumn{2}{|c|}{$\begin{array}{c}\text { Producción } \\
\text { de raíces }\end{array}$} \\
\hline & & & $\begin{array}{c}\text { Cantidad } \\
\left(\mathrm{N}^{\circ}\right)\end{array}$ & $\begin{array}{l}\text { Longitud } \\
(\mathrm{cm})\end{array}$ \\
\hline 1 & 40 & 40 & 2,5 & 3,6 \\
\hline 2 & 0 & 0 & 0,0 & 0,0 \\
\hline 3 & 60 & 0 & 0,0 & 0,0 \\
\hline 4 & 40 & 0 & 0,0 & 0,0 \\
\hline 5 & 60 & 20 & 5,0 & 6,9 \\
\hline 6 & 0 & 0 & 0,0 & 0,0 \\
\hline 7 & 100 & 60 & 2,3 & 7,1 \\
\hline 8 & 60 & 0 & 0,0 & 0,0 \\
\hline 9 & 80 & 60 & 4,3 & 4,3 \\
\hline 10 & 100 & 80 & 2,5 & 3,9 \\
\hline 11 & 100 & 20 & 2,0 & 1,8 \\
\hline 12 & 100 & 40 & 1,0 & 3,3 \\
\hline 13 & 40 & 40 & 2,5 & 7,1 \\
\hline 14 & 100 & 80 & 2,5 & 3,9 \\
\hline 15 & 100 & 40 & 4,5 & 4,8 \\
\hline 16 & 100 & 60 & 1,3 & 14,3 \\
\hline 17 & 60 & 20 & 1,0 & 7,0 \\
\hline 18 & 60 & 20 & 1,0 & 6,2 \\
\hline 19 & 20 & 20 & 1,0 & 6,8 \\
\hline 20 & 100 & 100 & 4,4 & 5,8 \\
\hline
\end{tabular}


con lo observado por Becker y Dautzenberg (14) para $N$. alpina, quienes obtuvieron tasas entre 2 y $13 \%$ de arraigamiento. Es probable, entonces, que la capacidad de arraigamiento de algunas especies del género Nothofagus esté condicionada genotípicamente.

Todas las estacas que sobrevivieron lograron formar raíces, observándose en promedio para el ensayo una tasa igual a $34 \%$ con 2,6 raíces de 5,8 $\mathrm{cm}$ de longitud por estaca. Esto concuerda, en parte, con lo señalado por Mebus (15) y Santelices y García (8).

En algunos clones se observó que la formación de callo fue superior a la tasa de arraigamiento, lo que reafirma que este proceso y el de rizogénesis son independientes.

Llama la atención la diferencia en la calidad de las raíces inducidas. En algunos casos, sobre todo por presentarse en baja cantidad, posteriormente no fue posible mantenerlas vivas. En forma posterior a que las plantas fueran acondicionadas y cultivadas durante un año en el vivero, la tasa final de sobrevivencia llegó a un $10 \%$.

Considerando que se utilizó una reducida cantidad de estacas por cada árbol madre, los resultados de este ensayo debieran ser validados estadísticamente, por lo que se sugiere continuar la investigación.

\section{CONCLUSIONES}

Existe una alta variabilidad en la tasa de arraigamiento y calidad de las raíces de $N$. alessandrii dependiendo del árbol madre de donde se colecta el material vegetal, razón por la cual el proceso de rizogénesis podría estar condicionado genotípicamente. Por ello, la selección de los árboles madres debería considerarse como un factor fundamental, sobre todo si se piensa realizar algún tipo de mejoramiento utilizando esta técnica.

Es posible obtener tasas de arraigamiento de $100 \%$ con material vegetal cosechado de plantas de no más de cuatro años de edad en el período de máximo crecimiento y tratadas con ácido indolbutírico al 0,5\% (p/p) de concentración. Para conseguirlo, es necesario trabajar en condiciones de invernadero; con una humedad ambiental no inferior a un $60 \%$; con camas calientes de arraigamiento y con aserrín esterilizado de $P$. radiata como substrato. Sin embargo, dependiendo del árbol madre de donde se cosechen las estacas, los resultados podrían llegar incluso a ser nulos.

En el proceso de rizogénesis de $N$. alessandrii no necesariamente debe ocurrir una secuencia en la formación de callo y raíces.

\section{BIBLIOGRAFIA}

(1) SAN MARTIN, J., H. FIGUEROA, C. RAMIREZ. Fitosociología de los bosques de ruíl (Nothofagus alessandrii Espinosa) en Chile Central. Revista Chilena de Historia Natural, 1984, vol. 57, p. 171-200.

(2) SERRA, M., R. GAJARDO, A. CABELLO. Programa de protección y recuperación de la flora nativa de Chile, Ficha Técnica de Especies Amenazadas: Nothofagus alessandrii Espinosa. Santiago, Chile: Corporación Nacional Forestal. 1986. 32 p.

(3) BENOIT, I. El libro rojo de la flora terrestre de Chile (Primera parte). Santiago, Chile: Corporación Nacional Forestal. 1989. 157 p.

(4) BUSTAMANTE, R., A. GREZ. Consecuencias ecológicas de la fragmentación de los bosques nativos. Ambiente y Desarrollo (Chile), 1995, vol. $11 \mathrm{~N}^{\circ} 2$, p. 58-63.

(5) DONOSO, C. Bosques templados de Chile y Argentina: variación, estructura y dinámica. Santiago, Chile: Editorial Universitaria, 1995, 483 p.

(6) HARTMANN, H., D. KESTER. Propagación de plantas, principios y prácticas. México: Ed. Continental, 1987, $760 \mathrm{p}$.

(7) DIRR, M., C. HEUSER. The reference manual of woody plant propagation: from seed to tissue culture. Georgia, U.S.A.: Varsity Press Inc., 1987, 239 p.

(8) SANTELICES, R., C. GARCIA. Efecto del ácido indolbutírico y la ubicación de la estaca en el rebrote de tocón sobre la rizogénesis de Nothofagus alessandrii Espinosa. Bosque (Chile), 2003, vol. 24, No 2, p. 53-61.

(9) SANTELICES, R. Propagación vegetativa de raulí, roble y coihue a partir de estacas. Ciencia e Investigación Forestal (Chile), 1993, vol. 7, No 1, p. 37-48.

(10) BÄRTELS, A. Gehölzvermehrung. Stuttgart, Deutschland: Verlag Eugen Ulmer. 1989. 370 p.

(11) WRIGHT, J. Mejoramiento genético de los árboles forestales. Estudios de silvicultura y productos forestales $\mathrm{N}^{\circ}$ 16. Roma, Italia: FAO. 1964. $436 \mathrm{p}$.

(12) KOZLOWSKI, T., P. KRAMER, S. PALLARDY. The physiological ecology of woody plants. San Diego, USA: Academic Press Inc., 1991, 657 p.

(13) ROJAS, P., P. ARCE, M. ARRIAGADA. Propagación vegetativa por estacas en Eucalyptus camaldulensis Dehn. Ciencia e Investigación Forestal (Chile), 1987, vol. 1, $\mathrm{N}^{\mathrm{o}} 2$, p. 1-8.

(14) BECKER, A. VON, H. DAUTZENBERG. Zur Stecklinsvermehrung bei Nothofagus procera (Poepp. et Endl) Oerst. Silvae Genetica, 1978, vol. 27, No 5, p. 178-183.

(15) MEBUS, I. Enraizamiento en estacas de Nothofagus spp. de la zona mesomórfica de Chile amenazadas de extinción. Tesis Licenciatura en Agronomía. Valdivia, Chile: Facultad de Ciencias Agrarias, Universidad Austral de Chile, 1993, 68 p. 